\title{
In Vivo Foveal Development Using Optical Coherence Tomography
}

\author{
Helena Lee, Ravi Purohit, Aarti Patel, Eleni Papageorgiou, Viral Sheth, Gail Maconachie, \\ Anastasia Pilat, Rebecca J. McLean, Frank A. Proudlock, and Irene Gottlob
}

University of Leicester Ulverscroft Eye Unit, Robert Kilpatrick Clinical Sciences Building, Leicester Royal Infirmary, Leicester, United Kingdom

Correspondence: Irene Gottlob, University of Leicester Ulverscroft Eye Unit, Robert Kilpatrick Clinical Sciences Building, PO Box 65, Leicester Royal Infirmary, Leicester LE2 7LX, UK;

ig15@leicester.ac.uk.

Submitted: January 26, 2015

Accepted: May 3, 2015

Citation: Lee H, Purohit R, Patel A, et al. In vivo foveal development using optical coherence tomography. Invest Opbthalmol Vis Sci. 2015;56:45374545. DOI:10.1167/iovs.15-16542
Purpose. To characterize the time course of normal foveal development in vivo in term infants and young children using handheld spectral-domain optical coherence tomography (HHSDOCT).

Methods. We obtained 534 HH-SDOCT scans from 261 infants, children, and young adults with a mean age of 4.9 years (range, 0-27 years). Each retinal layer was manually segmented in ImageJ and correlated with gestational age (GA) and visual acuity (VA). The developmental trajectories of each retinal layer at the fovea, parafovea, and perifovea were calculated using fractional polynomial modeling.

REsults. The central macular thickness (CMT) increases logarithmically between birth and 48.6 months GA. The foveal ganglion cell (GCL), inner plexiform, inner nuclear (INL), and outer plexiform layers decrease in thickness exponentially until 18 months GA. Interestingly, the parafoveal and perifoveal GCL and INL thicknesses initially decrease until 17 months GA and then increase in thickness until $65.5 \mathrm{GA}$. The foveal outer nuclear layer, inner segment, and outer segment of the photoreceptors increase in thickness logarithmically until 32.4, 26.9, and 45.3 months GA, respectively. The parafoveal and perifoveal outer retinal layers increase in thickness more gradually until 146 months GA. The thickness of the outer retinal layers and CMT were strongly correlated with VA, with $r=0.54(P<0.0001)$ and $r=0.52(P$ $<0.0001)$, respectively.

Conclusions. We have modeled for the first time the complex, nonlinear developmental trajectories for each retinal layer and demonstrate that development continues until adolescence. Our description of normal development will be helpful in diagnosing, monitoring, and understanding pediatric retinal disease.

Keywords: foveal development, retinal development, optical coherence tomography
$\mathrm{T}$ he human fovea is a specialized region of the retina of the eye, located in the center of the macula. It is responsible for the high spatial resolution and color vision experienced by healthy adult humans. ${ }^{1}$ It plays an important role in human visual cortex development, regulating calcarine fissure symmetry ${ }^{2}$ and cortical maps. ${ }^{3}$ The normal human retina is composed of several layers of neuronal cell bodies and synapses.

Optical coherence tomography (OCT) is a high-speed diagnostic imaging technique that provides high-resolution cross-sectional images of human retinal morphology in vivo. These images are obtained by measuring the echo time delay and magnitude of reflected or backscattered light from the retina. ${ }^{4} \mathrm{~A}$ high-resolution $(2.6 \mu \mathrm{m})$ handheld spectral-domain OCT (HH-SDOCT) suitable for pediatric retinal imaging has been recently developed and optimized for infants and young children. ${ }^{5,6}$ The retinal layers identified on OCT, including the retinal nerve fiber layer (RNFL), ganglion cell layer (GCL), inner plexiform layer (IPL), inner nuclear layer (INL), outer plexiform layer (OPL), outer nuclear layer (ONL), external limiting membrane (ELM), inner (IS) and outer (OS) segments of the photoreceptor layer, ellipsoid (ISE), contact cylinder (CC) of photoreceptors, and RPE have been correlated topographically with histology (Fig. 1). ${ }^{7,8}$ Each of these layers on OCT corresponds to its histologically named equivalent with the exception of the ONL and OPL. ${ }^{7}$ The axons of the photoreceptors (Henle fiber layer) that are part of the OPL on histology have similar reflectivity as the ONL on OCT. Therefore, the Henle fibers cannot be distinguished from the ONL and are seen as a single band. ${ }^{7}$ Handheld SDOCT has been shown to provide reliable retinal measurements in infants and small children, ${ }^{9}$ thus highlighting its potential to perform large-scale studies of retinal and foveal development in vivo.

Knowledge of development of the normal central retina and foveal structure has depended heavily on anatomic studies of the simian retina. ${ }^{10,11}$ Fewer observations have been made of the human fovea, with the number of eyes included in these studies ranging between 2 and 33 eyes. ${ }^{12-15}$ In addition, samples can be affected by tissue shrinkage as well as artifacts such as retinal detachment. Therefore, histology may not always represent in vivo morphology. As a result, it has not been possible to define with certainty the exact developmental trajectory and time course for each retinal layer at the fovea. Normal retinal development is complex and involves centrifugal displacement of the inner retinal layers away from the foveal center and centripetal displacement of the cone photoreceptors into the foveal center. ${ }^{12,16,17}$ Foveal development has been 

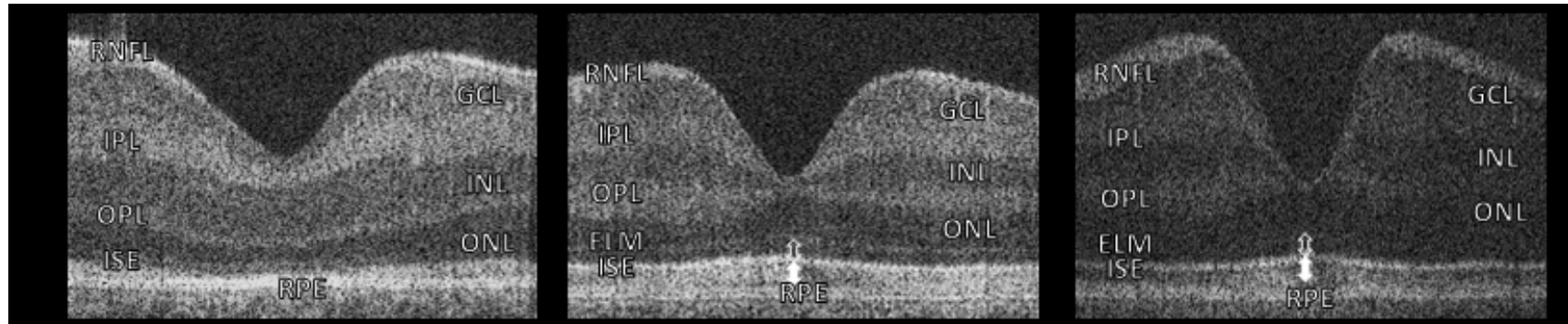

8.54 months

12.48 months
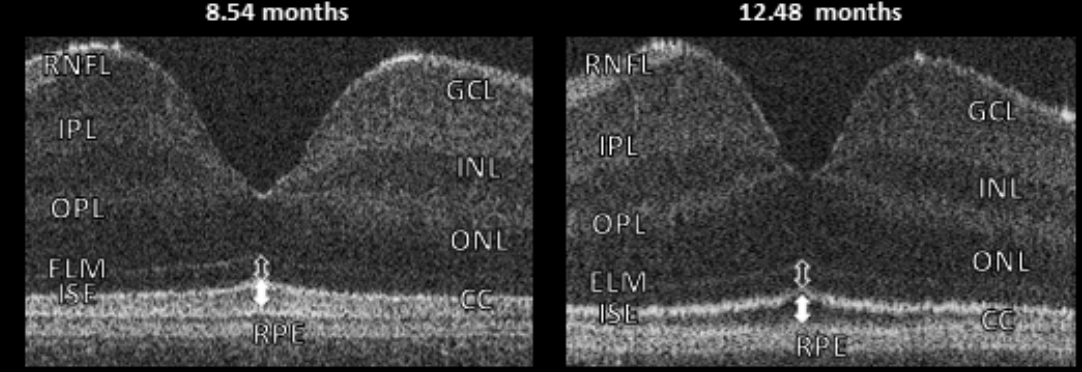

15.90 months

18.82 months

47.67 months

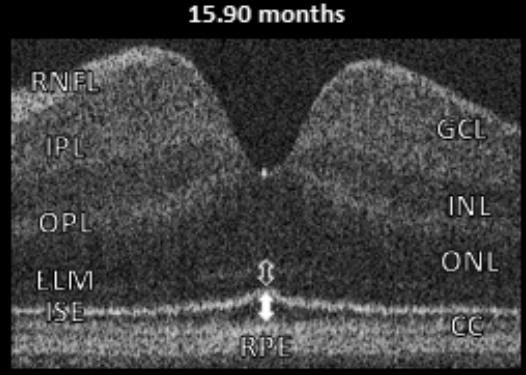

71.62 months

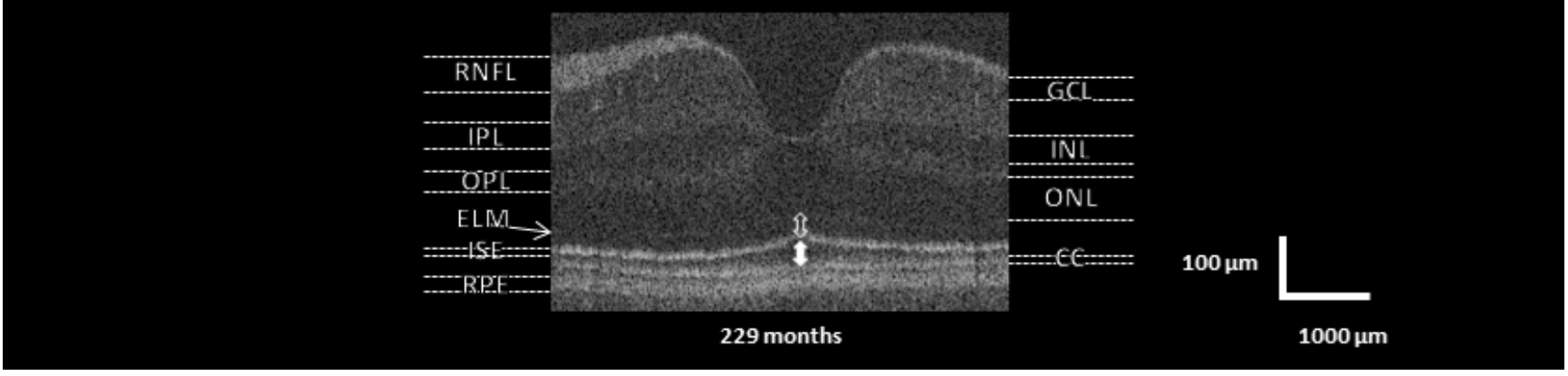

Figure 1. Foveal tomograms from between 8.54 months and 229 months GA illustrating retinal anatomy and features of foveal development. The ELM separates the ONL from the photoreceptor inner segment of the photoreceptors (empty block arrow) and the ISE separates the inner segment from the outer segment (white block arrow) of the receptors.

reported to be completed between 11 months and 5 years of age. ${ }^{12,13,18,19}$ In contrast, it has been shown that visual functions increase until adulthood, which has been attributed to maturation of the visual cortex. ${ }^{20}$

The aim of this study was to determine the developmental time course and trajectories for each retinal layer at the fovea and define the contribution of each retinal layer to the development of normal vision in full-term infants and young children using HH-SDOCT.

\section{MeTHODS}

\section{Participants}

The cohort for this study included 223 term children with a mean age of 2.2 years (range, 0-6.9 years) and 38 older children and adults with a mean age of 15 years (range, 7.127.0 years). Term was defined as a gestational age (GA) between 37 and 42 weeks. ${ }^{21}$ Participants were excluded if they had any preexisting ocular, neurological, or metabolic abnormalities that may affect eye development, a refractive error of +4.00 diopters (D) or greater in any axis, myopia of $-3.00 \mathrm{D}$ or greater in axis, or anisometropia of $1.5 \mathrm{D}$ or more. In addition, 24 further participants were recruited in whom there was either a failure to obtain a good-quality OCT scan in either eye $(n=20)$ or cystoid macular edema was identified directly after birth on their initial scans $(n=4)$. These scans were excluded from subsequent analysis. Images were deemed to be of sufficient quality for analysis if it was possible to segment the ONL and ISE and determine the central macular thickness (CMT) reliably. A total of 678 mixed cross-sectional and longitudinal tomograms were obtained, of which 534 (84.9\%) were of sufficient quality for analysis.

All participants underwent a full orthoptic and ophthalmologic examination, which included slit-lamp examination where possible, fundus examination, and measurement of visual acuity (VA). Visual acuity was assessed in younger infants and children by preferential looking using Teller acuity cards. Briefly in this procedure, the examiner, who is initially masked to the grating position, presents the card to the infant and judges the position of the gratings based on the response of the infant. The preferential looking test is based on infants' preference for patterned stimuli. In this test, a masked examiner presents a card with two apertures (one with grating patch of a known frequency and without a grating patch) to the infant and judges the position of the gratings based on the response of the infant. If it is correct, the examiner rotates the card 180 degrees and represents it. If a second correct response is obtained, the examiner proceeds to the next octave (i.e., a two-card ascension), until an error occurs on either presentation. When an error occurs, the examiner descends half an octave (i.e., a single card) and further trials are carried out in half-octave steps until a positive score is obtained. In cooperative children, Teller acuity cards and/or logMAR crowded optotypes (Glasgow Acuity Cards) were used to obtain VA.

Participants were divided into 16 age groups as follows: less than 40 weeks GA $(n=19), 41$ to 46 weeks GA $(n=18), 47$ to 
52 weeks GA $(n=7), 12$ to 14 months GA $(n=23), 15$ to 17 months GA $(n=24), 18$ to 20 months GA $(n=19), 21$ to 26 months GA $(n=15), 27$ to 32 months GA $(n=18), 33$ to 38 months GA $(n=14), 39$ to 44 months GA $(n=14), 45$ to 56 months GA $(n=24), 57$ to 68 months GA $(n=27), 69$ to 80 months GA $(n=17), 81$ to 92 months GA $(n=23), 93$ to 213 months GA $(n=22)$, and 214 to 309 months GA $(n=16)$. The demographic characteristics of the participants in this study are provided in Supplementary Tables S1 and S2.

\section{Optical Coherence Tomography}

Handheld SDOCT (Bioptigen Envisu System, Durham, NC, USA) was used to obtain a volumetric scan (consisting of 100 B-scans and 500 A-scans per B-scan) of the foveal region as previously described. ${ }^{9}$ All scans were carried out without the use of a speculum or sedation. To keep the children calm and cooperative for OCT examination, a variety of techniques were used. Infants and younger children were positioned sitting up on a parent's lap or supine cradled in a parent's arms. The OCT scanning of young infants was most often successful when acquired while bottle or breastfeeding. Older children responded well to age-appropriate animated fixation targets that used a portable laptop computer. All imaging was carried out in pairs by two of seven examiners (HL, RP, AP, EP, VS, GM, and RJM) of the HH-SDOCT system, which was set to continuous real-time scanning mode. One operator focused on carrying out the imaging while the second operator selected and captured the most optimal on-axis OCT scan. Scans were considered to be onaxis if the scan was centered in both the horizontal and vertical meridians and if there was no clipping evident on the tomograms. The acquired images were exported from the Bioptigen OCT software and imported into ImageJ software (http://imagej.nih.gov/ij/; provided in the public domain by the National Institutes of Health, Bethesda, MD, USA) where retinal layer segmentation was performed. The nomenclature used to label the segmented layers was based on previously established anatomical correlates with histology (Fig. 1).,22 If a border between two retinal layers could not be segmented reliably, then a combined measurement of the two layers was taken to optimize the reliability of the results obtained. ${ }^{9}$ This included the GCL and IPL, which together make the ganglion cell complex; the ONL and inner segment of the photoreceptors (IS), which together make the ONL-IS complex; and the outer segment of the photoreceptors (OS) and RPE, which make the (OS-RPE complex). For the purposes of this study, the parafovea and perifovea were defined as the regions measured at $1000 \mu \mathrm{m}$ and $2000 \mu \mathrm{m}$ from the central fovea, respectively.

\section{Statistics and Modeling}

We calculated the mean thickness measurement, SD, and $95 \%$ confidence interval for each retinal layer in each of the 16 age groups; the trajectory of development of each retinal layer over time; and the correlation between the thickness of each retinal layer and VA. Developmental trajectories were mapped out by carrying out fractional polynomial modeling. ${ }^{23}$ Fractional polynomial modeling is a very flexible method of modeling continuous, nonlinear data with skewed distributions. By choosing power transformations from the set $\{-2,-1,-0.5$, $0,0.5,1,2,3\}$, where 0 denotes the $\log$ transformation, fractional polynomial modeling can provide a range of shapes and curves that cannot be achieved using standard polynomials. To determine the relationship between retinal layer thickness measurements and Teller VA (measured in cycles per degree at $38 \mathrm{~cm}$ ), measurements from a single eye only from each examination visit was included in the analysis. If scans were available from both eyes from a single visit, then only the right eye measurements were included. First-order partial correlation coefficients were calculated between each retinal layer and Teller VA while controlling for age.

All analyses were considered significant at a type 1 probability value of $P$ less than 0.05 . Statistical analysis was performed by using Stata software (Copyright 1996-2014, Stata Corp., College Station, TX, USA).

The study adhered to the tenets of the Declaration of Helsinki and was approved by the local ethics committee. Informed consent was obtained from all parents/guardians of patients and control subjects participating in this study.

\section{Results}

\section{General Outline of Foveal Development}

The retinal tomograms obtained in this study confirm that human in vivo foveal development after birth consists of the dual processes of centrifugal displacement of the inner retinal layers and a centripetal displacement of the outer retinal layers, which occurs concurrently with photoreceptor elongation (Figs. 1, 2; Supplementary Movie S1). Individual retinal layers at the fovea and parafovea each have a specific developmental trajectory with some layers continuing to develop until 12 years of age (Supplementary Table S3).

\section{Changes in Retinal Thickness During Foveal Development}

The central retinal thickness at the fovea (CMT) increases logarithmically between birth and 48.6 months GA by $62.4 \%$ from a mean of $124.4 \mu \mathrm{m}$ to $203.70 \mu \mathrm{m}$ (Figs. 2A, 3A; Supplementary Table S4). In contrast, there is a smaller increase in the nasal (17\%) and temporal (21\%) parafoveal retinal thickness measurements (from means of 283.64-333.32 $\mu \mathrm{m}$ and 268.13-316.50 $\mu \mathrm{m}$, respectively, measured at $1000 \mu \mathrm{m}$ either side of the fovea) and nasal (6\%) and temporal (13\%) perifoveal measurements (from means of 294.54-302.45 $\mu \mathrm{m}$ and 254.5-274.31 $\mu \mathrm{m}$, respectively, measured at $2000 \mu \mathrm{m}$ either side of the fovea) by 48.6 months GA (Figs. 2A, 3A; Supplementary Tables S5, S6).

\section{Development of Inner Retinal Layers}

Regression of the inner retinal layers from the fovea is complete by 17.5 months GA (Figs. 2C, 3B). The GCL regresses from the fovea by 10.6 months GA. The IPL, INL, and OPL decrease in thickness exponentially, completing their regression from the fovea by 18.7, 17.6, and 17.8 months GA, respectively (Figs. 2E-I; 4B-E; Supplementary Table S3).

At the parafovea and perifovea, the developmental trajectories of the inner retinal layers are more complex than directly at the fovea. The RNFL and IPL decrease exponentially in thickness until 31.4 and 39 months GA, respectively (Figs. 2E, $2 \mathrm{G}, 4 \mathrm{~A}, 4 \mathrm{C})$. The GCL and INL initially decrease in thickness until 17 months GA and then increase in thickness until 65.5 months and 41.5 months GA, respectively (Figs. 2F, 2H, 4B, 4D). The OPL increases in thickness until 11.6 months GA (Figs. 2I, 4E).

The mean estimates for the inner retinal layers including the RNFL, GCL, IPL, INL, and OPL for each of our 16 predefined age groups at the fovea, parafovea, and perifovea are summarized in Supplementary Tables S4, S5, and S6.

\section{Development of Outer Retinal Layers}

In contrast to the inner retinal layers, the outer retinal layers at the fovea increase logarithmically by $185 \%$ from a mean 


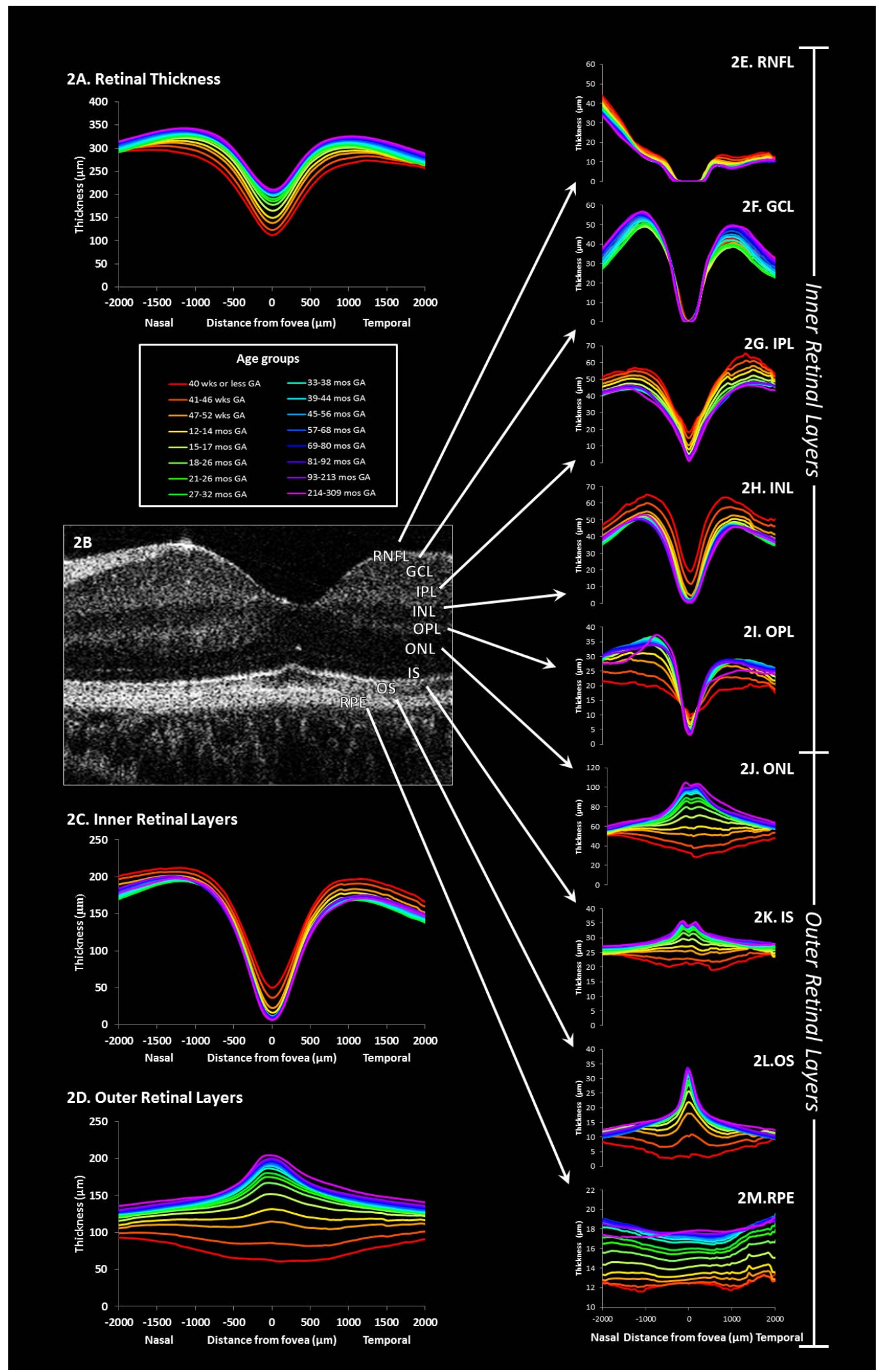

Figure 2. Mean thickness of each retinal layer plotted using a fourth-order polynomial fit for each of the 16 age groups that have been color coded. The inner retinal layers include the RNFL, GCL, IPL, INL, and OPL. The outer retinal layers include the ONL, IS, OS, and RPE. 

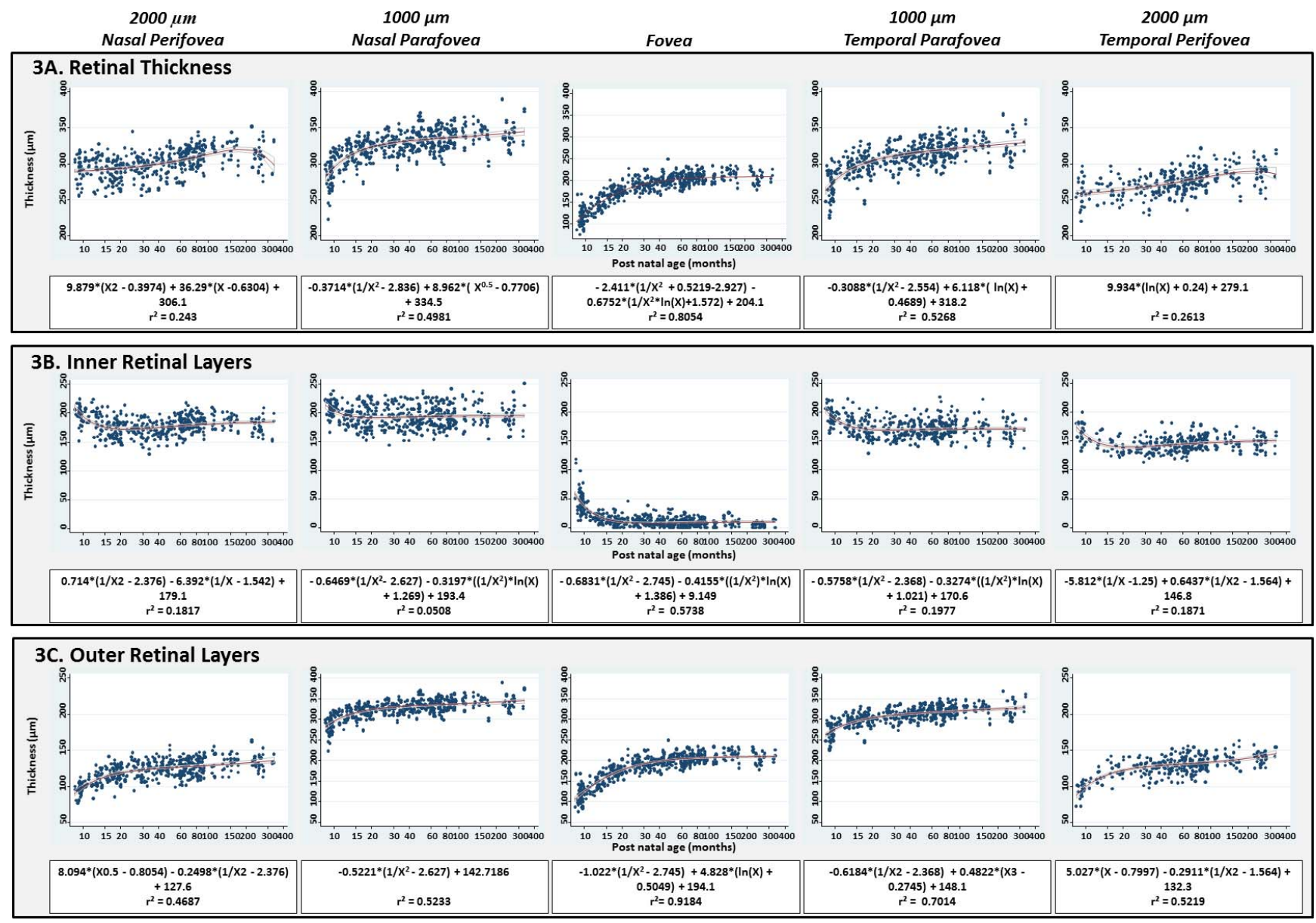

Figure 3. Development trajectories for the inner retinal layers, outer retinal layers, and total retinal thickness at the fovea, parafovea, and perifovea The parafoveal and perifoveal measurements were taken at $1000 \mu \mathrm{m}$ and $2000 \mu \mathrm{m}$ nasal and temporal to the central fovea. The trajectories have been plotted over an age range spanning 8.4 through 333.9 months GA. Each point represents a single examination value. The best fit curve line obtained with fractional polynomial regression analysis together with its $95 \%$ confidence interval is shown. The equation associated with the model is also given, where $X=G A$ in months $/ 100$.

thickness of 67.03 to $194.07 \mu \mathrm{m}$ between birth and 47 months GA, reaching maturity $(196.14 \mu \mathrm{m})$ by 75 months GA (Fig. 2D). The developmental trajectories for the outer retinal layers at the fovea and perifovea including the ONL, IS, OS, and RPE, and the mean estimates for each of our 16 predefined age groups at the fovea, parafovea, and perifovea are summarized in Figures 5A-D and in Supplementary Tables S4, S5, and S6.

The ONL, IS, and OS thicknesses increase logarithmically at the fovea between birth and 45.3, 26.9, and 32.4 months GA, respectively, from mean thicknesses of 34.37 to $95.57 \mu \mathrm{m}$ (ONL), 20.44 to $32.43 \mu \mathrm{m}$ (IS), and 3.06 to $30.79 \mu \mathrm{m}$ (OS). The parafoveal ONL, IS, and OS also increase in thickness logarithmically initially and then increase more gradually until 128.4, 29.8, and 146 months GA, respectively (Figs. 2J-M, 5AD).

The OS and ISE cannot be visualized in the central fovea in $62.1 \%$ and $34.8 \%$ of eyes aged between 37 and 40 weeks GA and 41 and 46 weeks GA, respectively. The OS was visible in the central fovea in all eyes from 47 weeks GA (Supplementary Table S7). Initially, the parafoveal and perifoveal photoreceptors are longer than the central photoreceptors. This difference reverses between 47 and 52 weeks GA, with the OS rapidly increasing in thickness at the fovea from a mean thickness of 3 $\mu \mathrm{m}$ at birth to $34 \mu \mathrm{m}$ by 44 months GA (987.3\%).

The appearance of the hyperreflective CC band (which is formed when the OS of the photoreceptors become sheathed by the underlying apical processes of the RPE) (Fig. 1) of the photoreceptors reflects foveal maturity. We found that this structure is rarely identifiable in the youngest age groups but is almost always visible after 47 months GA (Supplementary Table S8). The RPE increases in thickness at the fovea, parafovea, and perifovea between birth and 54.4 months GA and increases more slowly thereafter (Figs. 2M, 5D).

\section{Correlations With VA}

We identified a weak negative correlation between the thickness of the inner retinal layers with VA $(r=-0.16, P<$ 0.05). The thickness of the outer retinal layers and CMT were strongly correlated with Teller acuity $(r=0.54, P<0.0001$, and $r=0.52, P<0.0001$, respectively) (Supplementary Table S9). Each of the outer retinal layer thickness measurements were significantly correlated with Teller acuity. This includes the ONL $(r=0.51, P<0.0001)$, IS $(r=0.29, p<0.0001)$, OS $(r$ $=0.22, P<0.01)$, and RPE $(r=0.2, P<0.001)$ (Supplementary Table S9).

\section{Discussion}

To our knowledge, this is the first report of a large in vivo study of normal term human foveal development from birth to early adulthood. Previously, foveal development was thought to be 

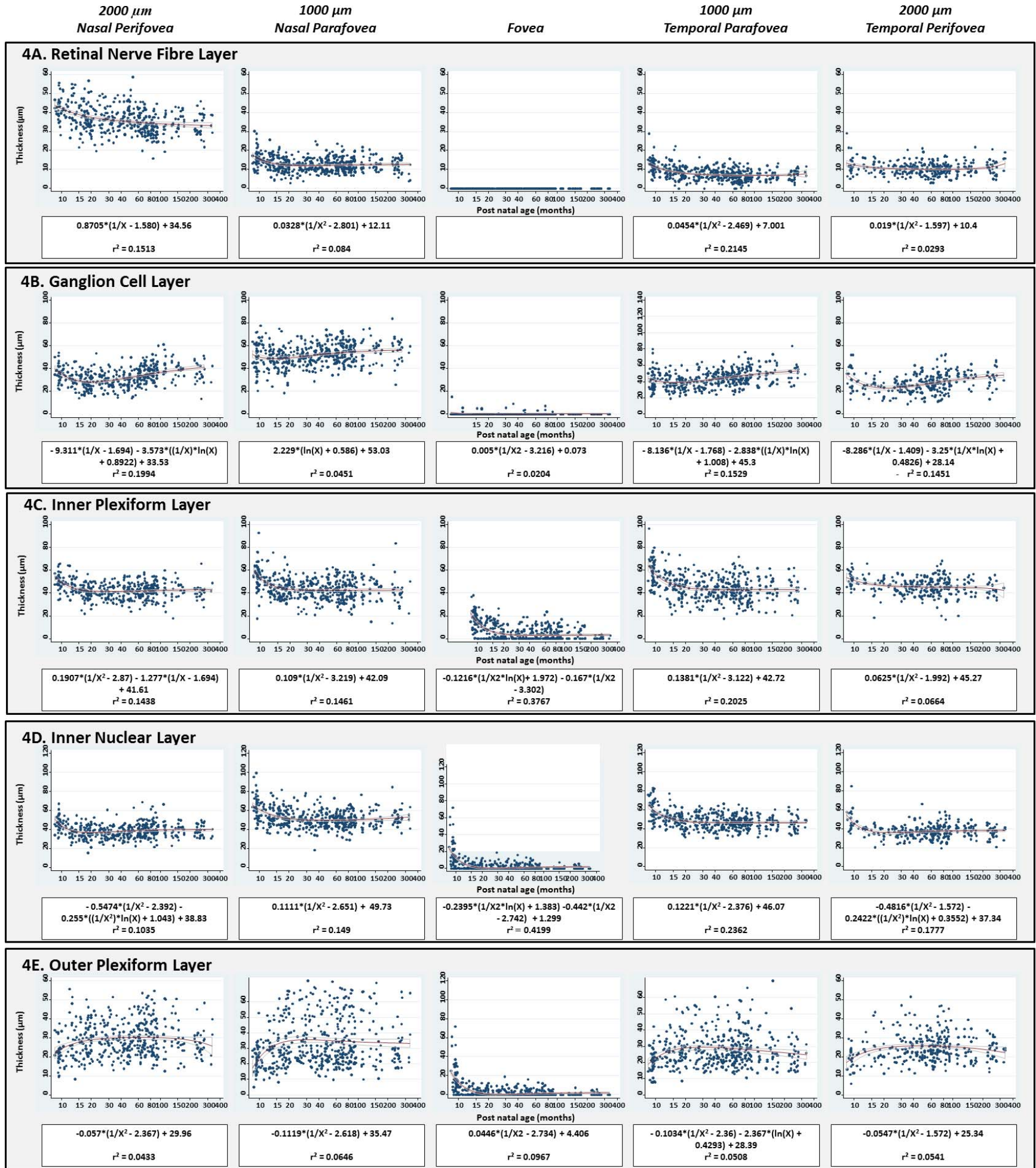

FIGURE 4. Development trajectories for the inner retinal layers: RNFL, GCL, IPL, INL, and OPL at the fovea, parafovea, and perifovea. The parafoveal and perifoveal measurements were taken at $1000 \mu \mathrm{m}$ and $2000 \mu \mathrm{m}$ nasal and temporal to the central fovea. The trajectories have been plotted over an age range spanning 8.4 through 333.9 months GA. Each point represents a single examination value. The best fit curve line obtained with fractional polynomial regression analysis together with its $95 \%$ confidence interval is shown. The equation associated with the model is also given, where $X=G A$ in months $/ 100$

complete by 5 years of age. ${ }^{12}$ We demonstrate that foveal development continues for much longer, at least until 12 years of age.

There have been only two previous in vivo OCT studies of human foveal development. This included a study of foveal development after premature birth, which included 4 term infants aged between 1 and 9 months and 4 children aged between 2 and 5 years, and a second study that described human foveal maturation using OCT and histology, which included 30 term infants and 16 children. ${ }^{5,8}$ The developmen- 

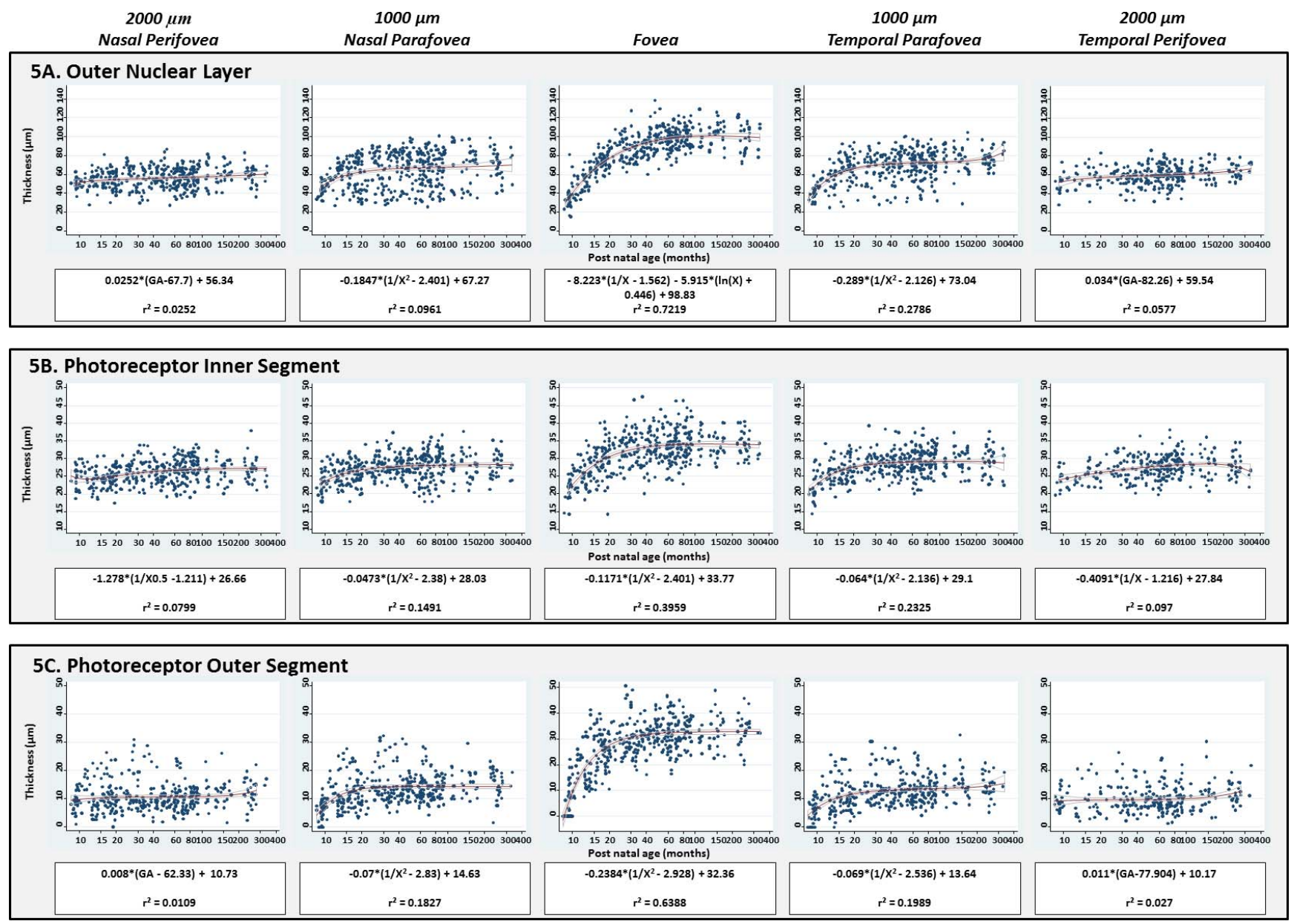

\section{D. Retinal Pigment Epithelium}
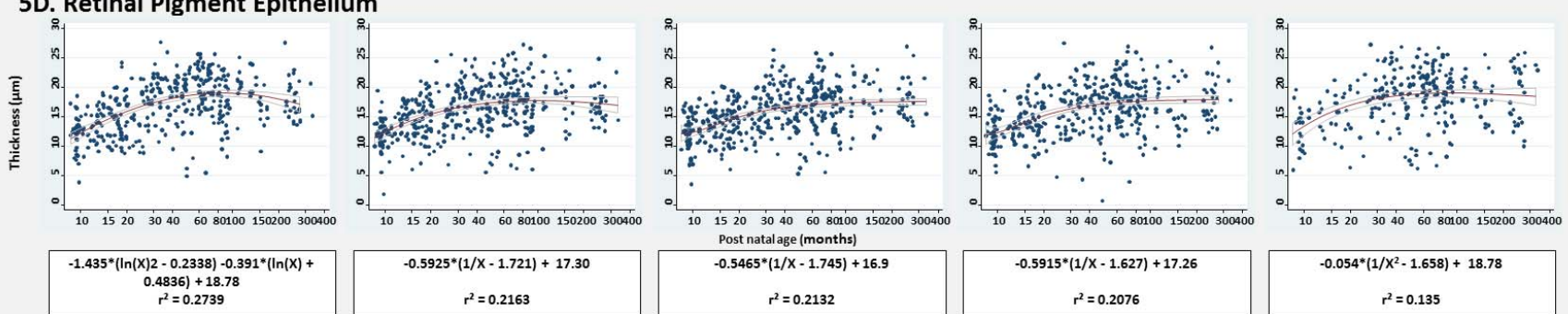

FiguRE 5. Development trajectories for the ONL, IS, OS, and RPE at the fovea, parafovea, and perifovea. The parafoveal and perifoveal measurements were taken at $1000 \mu \mathrm{m}$ and $2000 \mu \mathrm{m}$ nasal and temporal to the central fovea. The trajectories have been plotted over an age range spanning 8.4 through 333.9 months GA. Each point represents a single value from each OCT examination. The best fit curve line obtained with fractional polynomial regression analysis together with its $95 \%$ confidence interval is shown. The equation associated with the model is also given, where $X=G A$ in months $/ 100$

tal trajectories described in this study follows on directly from those described previously in premature infants. ${ }^{5,8} \mathrm{~A}$ direct comparison of the mean retinal layer thickness values reported in our study with those previously reported is limited by the differences in the age ranges used to calculate mean retinal layer thickness values. For example, in the study by Maldonado et al., ${ }^{5}$ a mean value of $10 \mu \mathrm{m}$ for inner retinal layer thickness was reported for infants aged between 1 and 9 months. In our study, we divided this age range into four separate groups and obtained mean inner retinal layer thickness values of 37.85 , $22.93,17.88$, and $13.92 \mu \mathrm{m}$ at 41 to 46 weeks, 27 to 52 weeks, 12 to 14 months, and 15 to 17 months GA, respectively. This illustrates the extent to which age can affect retinal layer thickness measurements. Another important difference is in the definitions of which layers are included as part of the inner and outer retinal layers in each study. In our study, we included the OPL as part of the inner retinal layers, whereas in the study by Maldonado et al., ${ }^{5}$ the OPL was included as part of the outer retinal layers. It would be important in the future to agree to standard definitions for each of the retinal layers and the age ranges to be used when reporting pediatric normative OCT data to ensure that all measurements are consistent and comparable across studies.

Regression of the inner retinal layers from the fovea has been described to be complete at between 6 weeks and 9 months postnatally. ${ }^{19}$ It is hypothesized that mechanical stretching of the retina caused by growth of the eye drives migration of the inner retinal layers away from the fovea. ${ }^{24}$ The 
largest change in axial length (reflecting eye growth) occurs during the first 24 months of life, when the axial length reaches $90 \%$ of mean adult values. ${ }^{24-26}$ We found that the timing of inner retinal layer regression from the fovea is consistent with the mechanical stretch hypothesis, occurring in parallel with fast eye growth and reaching completion by 17.5 months GA.

Interestingly, the developmental trajectories of the inner retinal layers at the parafovea and perifovea are much more dynamic than directly at the fovea. In the case of the GCL and INL, we observed initial decreases in retinal layer thickness followed by subsequent increases. This developmental pattern may represent the dual processes of migration of the inner retinal layers away from the fovea and their thickening with age. These two processes occur at different rates for each inner retinal layer, resulting in the unique developmental trajectories obtained for each retinal layer.

Histological studies have demonstrated that cone-packing density at the fovea only reaches half adult values at 45 months, suggesting that development may continue until adulthood. ${ }^{13}$ In support of this hypothesis, we found that the ONL continues to increase in thickness at least until 12 years of age, which may reflect ongoing increases in the number of foveal cone photoreceptors.

Consistent with previous reports, we found that the OS and ISE cannot always be visualized in the central fovea before 46 weeks GA. ${ }^{5,8}$ This contrasts with histological findings, where the IS and OS can always be visualized at the fovea from birth in term infants. The IS and OS may be too short and immature perinatally to form a visible band on OCT. $^{8}$ Possibly, the photoreceptors are undergoing centripetal migration and elongation at different rates in individual infants, explaining why bands corresponding to the IS and OS on OCT are not visible in all newborns at the central fovea.

The presence of the hyperreflective contact cylinder band is a reflection of foveal maturity. ${ }^{5,8}$ We found that the $\mathrm{CC}$ is rarely identifiable in the youngest age groups but is almost always visible after 47 months GA. This is consistent with previous reports that suggest that the $\mathrm{CC}$ is identifiable between 24 months and 5 years. ${ }^{8}$ In contrast, in premature infants, the CC was not visible in any infants younger than 10 years. ${ }^{5}$ This suggests that development of the outer retinal layers may differ between premature and term children on OCT. Larger-scale longitudinal OCT studies of premature foveal development would be needed to resolve these differences.

Interestingly, we show that the RPE increases in thickness until 54.4 months GA. This may be essential to normal visual function and development, as it is known that atrophy of the RPE occurs in diseases of the retina, such as AMD and retinal dystrophies. ${ }^{27}$

In adults with albinism, the length of the OS is strongly correlated with VA. ${ }^{28}$ Therefore, we investigated if a similar correlation between measurements of retinal layer thickness at the fovea and VA could be identified in the developing child. The outer retinal layers have the most significant contributions to the development of VA, with the strongest correlation occurring with the ONL. Visual functions, such as hyperacuity, continue to develop until 21 years of age. ${ }^{20}$ Although this has been attributed to refinement of neural circuitry at the cortical level, it is possible that ongoing retinal development during this period also may be contributing to this process.

It is important to note that in addition to age-related differences in retinal layer thickness measurements, there is also a significant amount of variation among participants within the same age group. One possible explanation for this is that this range of variation is normal and to be expected, as this phenomenon also has been described in other neurodevelopmental studies; for example, describing maturation of motor function in children. ${ }^{29-32}$ Further supporting evidence for this is found in other OCT studies providing normative adult data. ${ }^{33-35}$ In these studies, the SDs reported for CMT were 14.5 $\mu \mathrm{m},{ }^{35} 22.5 \mu \mathrm{m},{ }^{33}$ and $16.81 \mu \mathrm{m} .{ }^{34}$ This is consistent with the SD of $13.83 \mu \mathrm{m}$ calculated for CMT in our adult group.

Another possibility is that the accuracy of segmentation also may be contributing to the variation in retinal layer thickness measurements observed, which we had previously demonstrated in a previous test-retest study of the reliability of the HH-SDOCT. ${ }^{9}$ In that study, the foveal OPL had an intraclass correlation coefficient of 0.401 , which suggests that a proportion of the degree of variability that we observe in this current study of the OPL measurements may be partly attributable to inaccuracies in segmentation. It is likely that each of these factors contributes to some degree to the variation seen in the measurements obtained.

We have demonstrated that foveal development is a complex, nonlinear process that continues after the age of 5 years into adolescence, mimicking findings of longitudinal magnetic resonance imaging brain development studies. ${ }^{36}$ Unique patterns of migration and elongation define development of each retinal layer. Our description of normal development will be helpful in detecting, monitoring, and understanding pathological processes that effect retinal and visual development in infants and young children.

\section{Acknowledgments}

Supported by the Medical Research Council, London, United Kingdom (Grant MR/J004189/1), Ulverscroft Foundation, Leicester, United Kingdom, The National Eye Research Centre, and Nystagmus Network United Kingdom.

Disclosure: H. Lee, None; R. Purohit, None; A. Patel, None; E. Papageorgiou, None; V. Sheth, None; G. Maconachie, None; A. Pilat, None; R.J. Mclean, None; F.A. Proudlock, None; I. Gottlob, None

\section{References}

1. Rossi EA, Roorda A. The relationship between visual resolution and cone spacing in the human fovea. Nat Neurosci. 2010;13: 156-157.

2. Neveu MM, von dem Hagen E, Morland AB, et al. The fovea regulates symmetrical development of the visual cortex. $J$ Comp Neurol. 2008;506:791-800.

3. Baseler HA, Brewer AA, Sharpe LT, et al. Reorganization of human cortical maps caused by inherited photoreceptor abnormalities. Nat Neurosci. 2002;5:364-370.

4. Gabriele ML, Wollstein G, Ishikawa H, et al. Optical coherence tomography: history, current status, and laboratory work. Invest Ophthalmol Vis Sci. 2011;52:2425-2436.

5. Maldonado RS, O'Connell RV, Sarin N, et al. Dynamics of human foveal development after premature birth. Ophthalmology. 2011;118:2315-2325.

6. Maldonado RS, Izatt JA, Sarin N, et al. Optimizing hand-held spectral domain optical coherence tomography imaging for neonates, infants, and children. Invest Ophthalmol Vis Sci. 2010;51:2678-2685.

7. Spaide RF, Curcio CA. Anatomical correlates to the bands seen in the outer retina by optical coherence tomography: literature review and model. Retina. 2011;31:1609-1609.

8. Vajzovic L, Hendrickson AE, O'Connell RV, et al. Maturation of the human fovea: correlation of spectral-domain optical coherence tomography findings with histology. Am J Ophthalmol. 2012;154:779-789.e2

9. Lee H, Proudlock F, Gottlob I. Is handheld optical coherence tomography reliable in infants and young children with and 
without nystagmus? Invest Ophthalmol Vis Sci. 2013;54: 8152-8159.

10. Dorn EM, Hendrickson L, Hendrickson AE. The appearance of rod opsin during monkey retinal development. Invest Ophthalmol Vis Sci. 1995;36:2634-2651.

11. Hendrickson A. A morphological comparison of foveal development in man and monkey. Eye (Lond). 1992;6:136144.

12. Hendrickson AE, Yuodelis C. The morphological development of the human fovea. Ophthalmology. 1984;91:603-612.

13. Yuodelis C, Hendrickson A. A qualitative and quantitative analysis of the human fovea during development. Vision Res. 1986;26:847-855.

14. Linberg KA, Fisher SK. A burst of differentiation in the outer posterior retina of the eleven-week human fetus: an ultrastructural study. Vis Neurosci. 1990;5:43-60.

15. Hendrickson A, Possin D, Vajzovic L, et al. Histologic development of the human fovea from midgestation to maturity. Am J Ophthalmol. 2012;154:767-778.e2.

16. Provis JM, Hendrickson AE. The foveal avascular region of developing human retina. Arch Ophthalmol. 2008;126:507511.

17. Provis JM, Diaz CM, Dreher B. Ontogeny of the primate fovea: a central issue in retinal development. Prog Neurobiol. 1998; 54:549-580.

18. Abramov I, Gordon J, Hendrickson A, et al. The retina of the newborn human infant. Science. 1982;217:265-267.

19. Dubis AM, Costakos DM, Subramaniam CD, et al. Evaluation of normal human foveal development using optical coherence tomography and histologic examination. Arch Ophthalmol. 2012;130:1291-1300.

20. Wang YZ, Morale SE, Cousins R, et al. Course of development of global hyperacuity over lifespan. Optom Vis Sci. 2009;86: 695-700.

21. Spong CY. Defining "term" pregnancy: recommendations from the Defining "Term" Pregnancy Workgroup. JAMA. 2013;309:2445-2446.

22. Drexler W, Sattmann H, Hermann B, et al. Enhanced visualization of macular pathology with the use of ultrahighresolution optical coherence tomography. Arch Ophthalmol. 2003;121:695-706.

23. Royston P, Sauerbrei W. A new approach to modelling interactions between treatment and continuous covariates in clinical trials by using fractional polynomials. Stat Med. 2004; 23:2509-2525.

24. Springer AD, Hendrickson AE. Development of the primate area of high acuity, 3: temporal relationships between pit formation, retinal elongation and cone packing. Vis Neurosci. 2005;22:171-185.

25. Cook A, White S, Batterbury M, et al. Ocular growth and refractive error development in premature infants with or without retinopathy of prematurity. Invest Ophthalmol Vis Sci. 2008;49:5199-5207.

26. Gordon RA, Donzis PB. Refractive development of the human eye. Arch Ophthalmol. 1985;103:785-789.

27. Michaelides M, Hunt DM, Moore AT. The genetics of inherited macular dystrophies. J Med Genet. 2003;40:641-650.

28. Thomas MG, Kumar A, Mohammad S, et al. Structural grading of foveal hypoplasia using spectral-domain optical coherence tomography a predictor of visual acuity? Ophthalmology. 2011;118:1653-1660

29. Denckla MB. Development of speed in repetitive and successive finger-movements in normal children. Dev Med Child Neurol. 1973;15:635-645.

30. Denckla MB. Development of motor co-ordination in normal children. Dev Med Child Neurol. 1974;16:729-741.

31. Wolff PH, Gunnoe CE, Cohen C. Associated movements as a measure of developmental age. Dev Med Child Neurol. 1983; 25:417-429.

32. Wolff PH, Gunnoe C, Cohen C. Neuromotor maturation and psychological performance: a developmental study. Dev Med Child Neurol. 1985;27:344-354.

33. Grover S, Murthy RK, Brar VS, et al. Normative data for macular thickness by high-definition spectral-domain optical coherence tomography (Spectralis). Am J Ophthalmol. 2009; 148:266-271.

34. Huang J, Liu X, Wu Z, et al. Macular thickness measurements in normal eyes with time-domain and Fourier-domain optical coherence tomography. Retina. 2009;29:980-987.

35. Liu T, Hu AY, Kaines A, et al. A pilot study of normative data for macular thickness and volume measurements using Cirrus high-definition optical coherence tomography. Retina. 2011; 31:1944-1950.

36. Giedd JN, Blumenthal J, Jeffries NO, et al. Brain development during childhood and adolescence: a longitudinal MRI study. Nat Neurosci. 1999;2:861-863. 